\title{
Aplicación de un Ciclo de Mejora en el Aula en la asignatura Estomatología y Patología Sistémica: el aprendizaje a través del role-play on-line
}

\section{Application of a Classroom Improvement Cycle in the subject Stomatology and Systemic Pathology: learning through online role-play}

María del Carmen Machuca Portillo

Universidad de Sevilla

Departamento Estomatología

mmachuca@us.es

https://orcid.org/0000-0001-8220-5605

DOI: http://dx.doi.org/10.12795/9788447231003.043

Pp.: 903-924 


\section{Contexto}

El Ciclo de Mejora en el Aula (CIMA) se ha desarrollado en la asignatura "Estomatología y Patología Sistémica" -que se imparte en el tercer curso del Grado en Odontología de la Universidad de Sevilla (US)-, siguiendo las directrices y contenidos del programa de Formación e Innovación Docente del Profesorado (FIDOP) centrado en la práctica en el aula (Porlán, 2017).

La asignatura "Estomatología y Patología Sistémica" será abreviadamente referida en el texto como asignatura "EP Sistémica". Dicha asignatura se impartió por primera vez en el curso académico 2011-2012, según el cuarto Plan de Estudios de Graduado en Odontología (Resolución de la Universidad de Sevilla, de 20 de diciembre de 2010, BOE de 20 de Enero de 2011). Es una asignatura de reciente creación que no existía en la Licenciatura de Odontología y, desde sus inicios, la metodología docente y la evaluación por competencias ha estado adaptada al proceso de enseñanza-aprendizaje tipo Bolonia.

Es una asignatura obligatoria, de primer cuatrimestre, que consta de 6 créditos presenciales ( 60 horas), divididos en 2 créditos teóricos, 1 crédito preclínico y 2 créditos clínicos y desde sus inicios ha sido coordinada por la profesora María del Carmen Machuca Portillo.

El objetivo de la asignatura es que el alumnado conozca las manifestaciones orales de las enfermedades sistémicas, que pueda identificar y diagnosticar la patología bucodental en pacientes con patología sistémica, identificar los fármacos, y diagnosticar urgencias médicas en el gabinete dental.

Ciclos de Mejora en el Aula (2020). Experiencias de Innovación Docente de la US Esta obra se distribuye con la licencia Creative Commons 
El presente ciclo de mejora se diseñó para realizarse con total presencialidad, tanto en aula, como en laboratorio y en la clínica dental de la Facultad de Odontología. Por motivos de la pandemia por la Covid-19, el curso académico comenzó con un Plan de Contingencia. Este planteaba una menor actividad académica presencial como consecuencia de medidas sanitarias de distanciamiento interpersonal, que limitan el aforo permitido en las aulas y, posteriormente, se suspendió la actividad presencial. Por ello las clases teóricas y preclínicas se impartieron por videoconferencia on-line no presencial en la plataforma Blackboard Collaborate Ultra.

Los sujetos del estudio han sido los 98 alumnos de la Facultad de Odontología de la Universidad de Sevilla de tercer curso, matriculados en la asignatura Estomatología y Patología Sistémica del Grado en Odontología en el curso académico 2020-2021. No han tenido experiencia previa en otros ciclos de mejora ni han participado en proyectos de innovación docente.

\section{Diseño del Ciclo de Mejora en el Aula}

Seleccionada la asignatura (EP Sistémica) en la que se llevaría a cabo el ciclo de mejora y, al tener autonomía para realizar las adaptaciones de la docencia al ser coordinadora de la misma, se identificaron los temas/contenidos del temario (Historia y Exploración Clínica en Estomatología y Patología Sistémica) y se planificó la temporalización de la docencia (del 22 de octubre al 26 de noviembre del 2020).

Ciclos de Mejora en el Aula (2020). Experiencias de Innovación Docente de la US Esta obra se distribuye con la licencia Creative Commons 


\section{Mapa de contenidos y problemas claves}

El tema seleccionado fue Historia y Exploración Clínica en Estomatología y Patología Sistémica. Resumidamente queda esquematizado (como se aprecia en la Figura 1) con un problema central que es "El dolor de muelas y la Caries Dental". Y, a partir del mismo, el alumnado debe realizar un camino que es la HISTORIA CLÍNICA, con todas sus múltiples y complejas ramificaciones (anamnesis, examen físico, resumen, problemas de salud, plan diagnóstico y plan terapéutico), debiendo ser muy exhaustivo con todas las ramificaciones de la anamnesis. El alumnado también debe seguir un segundo camino que es la EXPLORACÍ́N ODONTOLÓGICA, en todas sus aseveraciones (intraoral y extraoral). Y puede ser necesario un tercer camino que son las PRUEBAS COMPLEMENTARIAS.

Todos estos conocimientos y conceptos están afectados por la situación de alerta sanitaria provocada por la pandemia por la Covid-19, y deben ser conocidos por el alumnado.

Destaca como fondo del mapa, la HUMANIZACIÓN DE LA ASISTENCIA, es decir, la importancia de ver enfermos y no enfermedades y tratarlos con respeto y dignidad.

Ciclos de Mejora en el Aula (2020). Experiencias de Innovación Docente de la US Esta obra se distribuye con la licencia Creative Commons 


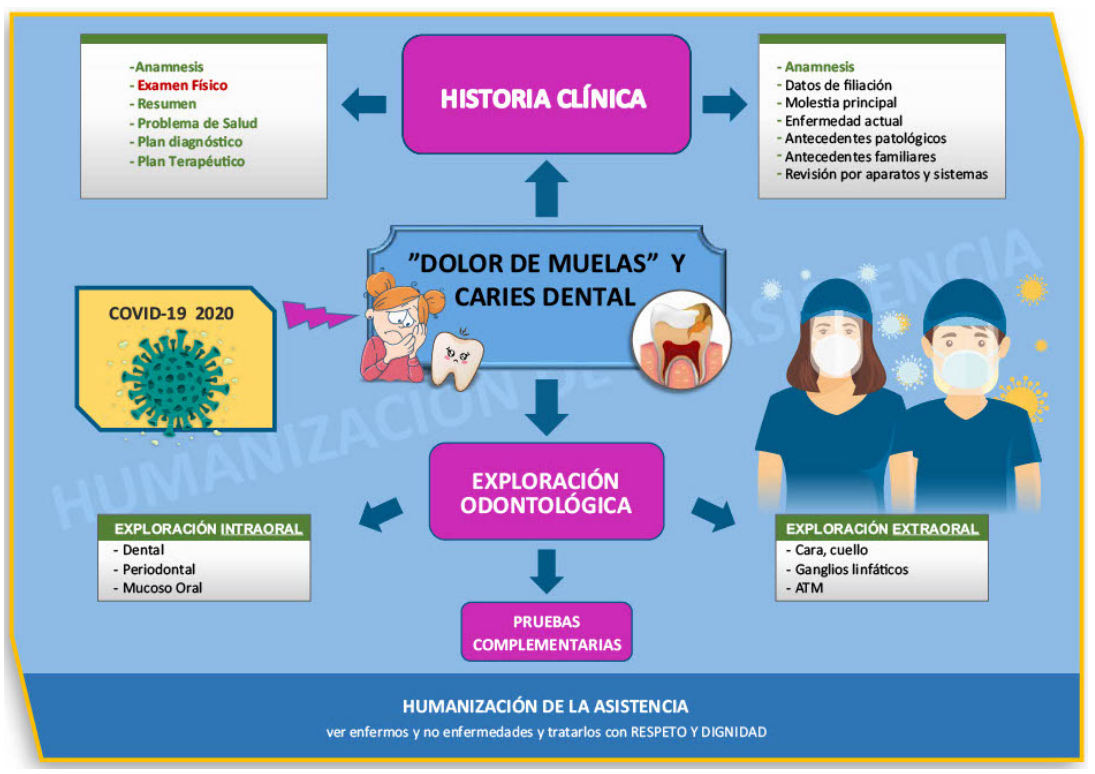

Figura 1. Mapa de contenidos.

\section{Modelo metodológico y secuencia de actividades programadas}

El modelo metodológico posible, basado en la reelaboración de las ideas de los estudiantes, queda representado en la Figura 2.

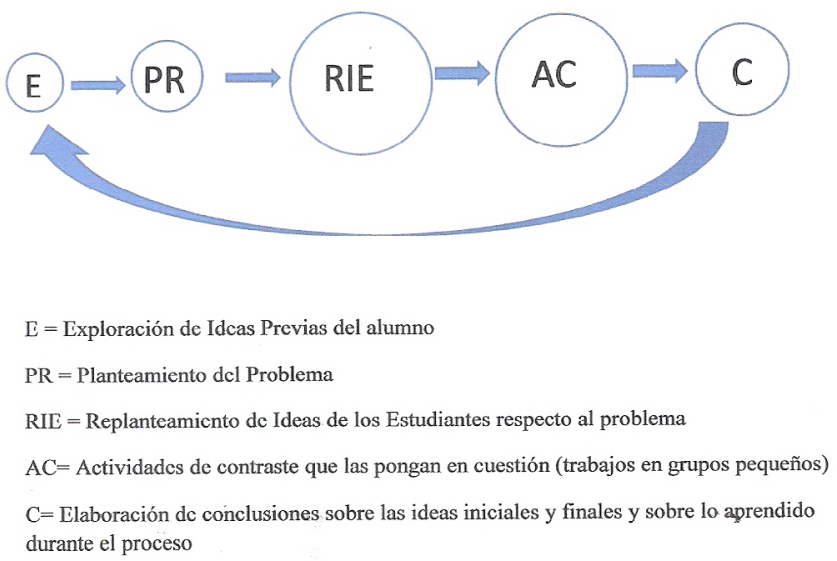

Figura 2. Modelo metodológico.

Ciclos de Mejora en el Aula (2020). Experiencias de Innovación Docente de la US C) Esta obra se distribuye con la licencia Creative Commons Reconocimiento-NoComercial-SinObraDerivada Internacional (CC BY-NC-ND 4.0.) 
La secuencia de actividades queda descrita en las siguientes tablas:

SESIÓN 1: DOCENCIA - CRÉDITOS TEÓRICOS. 22 octubre 2020

\begin{tabular}{|l|l|l|c|}
\hline $\begin{array}{l}\text { DESCRIPCIÓN } \\
\text { FASE DEL MODELO METOD. }\end{array}$ & FINALIDAD & RECURSO & T \\
\hline $\begin{array}{l}\text { Rellenar individualmente } \\
\text { un cuestionario inicial } \\
\text { con 6 preguntas del } \\
\text { tema sobre el que se va } \\
\text { a desarrollar el CIMA. Lo } \\
\text { rellenan y lo envían por } \\
\text { mail durante la hora de } \\
\text { la clase } \\
\text { E }\end{array}$ & $\begin{array}{l}\text { Conocer las ideas } \\
\text { previas que tienen } \\
\text { el tema que se va a }\end{array}$ & $\begin{array}{l}\text { Cuestionario } \\
\text { de Ideas } \\
\text { Previas }\end{array}$ & $15^{\prime}$ \\
\end{tabular}

SESIÓN 2: DOCENCIA - CRÉDITOS PRECLÍNICOS. 5 noviembre 2020

\begin{tabular}{|l|l|l|c|}
\hline DESCRIPCIÓN & FINALIDAD & RECURSOS & T \\
FASE DEL MODELO METOD. & & & \\
\hline $\begin{array}{l}\text { Planteamiento de casos } \\
\text { problemas } \\
\text { Se presentan seis casos } \\
\text { clinicos con imágenes y } \\
\text { muy pocos datos para } \\
\text { que los alumnos en } \\
\text { grupos pequeños puedan } \\
\text { "crearlos" y realizar un } \\
\text { diagnóstico } \\
\text { PR }\end{array}$ & $\begin{array}{l}\text { Enicio con algo que } \\
\text { resulte de interés. }\end{array}$ & $\begin{array}{l}\text { Fotografías y } \\
\text { radiografías }\end{array}$ & 10' \\
\hline
\end{tabular}

Ciclos de Mejora en el Aula (2020). Experiencias de Innovación Docente de la US Esta obra se distribuye con la licencia Creative Commons 


\begin{tabular}{|c|c|c|c|}
\hline $\begin{array}{l}\text { Trabajo en Grupo: Idea de } \\
\text { Alumnos } \\
\text { Cada Grupo de alumnos } \\
\text { (A y B) se subdividió en } 6 \\
\text { grupos de } 8 \text { alumnos y se } \\
\text { trabajó en la plataforma } \\
\text { Collaborate Ultra } \\
\text { RIE }\end{array}$ & $\begin{array}{l}\text { Durante esta } \\
\text { actividad el } \\
\text { alumno realizó una } \\
\text { descripción del } \\
\text { caso clínico, historia } \\
\text { clínica y exploración. } \\
\text { Cada grupo de } 8 \\
\text { alumno realiza un } \\
\text { informe que lo } \\
\text { entrega por mail }\end{array}$ & $\begin{array}{l}\text { Trabajo } \\
\text { en grupos } \\
\text { pequeños en } \\
\text { la plataforma } \\
\text { Collaborate } \\
\text { Ultra }\end{array}$ & $90^{\prime}$ \\
\hline $\begin{array}{l}\text { Resolución de Dudas } \\
\text { AC }\end{array}$ & $\begin{array}{l}\text { El profesorado } \\
\text { ayuda a los } \\
\text { diferentes grupos } \\
\text { de alumnos durante } \\
\text { toda la sesión, } \\
\text { aclarando dudas } \\
\text { y orientándolos. } \\
\text { Va entrando en } \\
\text { cada grupo de la } \\
\text { plataforma }\end{array}$ & $\begin{array}{l}\text { Asesoramiento } \\
\text { del } \\
\text { profesorado y } \\
\text { orientación }\end{array}$ & $90^{\prime}$ \\
\hline $\begin{array}{l}\text { Puesta en común de } \\
\text { los informes de cada } \\
\text { grupo-Intervención del } \\
\text { alumnado } \\
\text { C }\end{array}$ & $\begin{array}{l}\text { El portavoz de } \\
\text { cada grupo expone } \\
\text { resumidamente el } \\
\text { caso clínico con sus } \\
\text { aportaciones más } \\
\text { relevantes }\end{array}$ & $\begin{array}{l}\text { Informes de } \\
\text { los grupos }\end{array}$ & $10^{\prime}$ \\
\hline $\begin{array}{l}\text { Puesta en común de } \\
\text { los informes de cada } \\
\text { grupo Intervención del } \\
\text { profesorado } \\
\text { C }\end{array}$ & $\begin{array}{l}\text { Cada profesor } \\
\text { comenta lo más } \\
\text { relevante de cada } \\
\text { caso clínico y de } \\
\text { los informes y se } \\
\text { resumen todas las } \\
\text { intervenciones y lo } \\
\text { más relevante }\end{array}$ & & $10^{\prime}$ \\
\hline
\end{tabular}

SESIÓN 3: DOCENCIA-CRÉDITOS PRECLÍNICOS. 12 noviembre 2020

\begin{tabular}{|l|l|l|l|}
\hline $\begin{array}{l}\text { DESCRIPCIÓN } \\
\text { FASE DEL MODELO METOD. }\end{array}$ & FINALIDAD & RECURSOS & T \\
\hline
\end{tabular}

Ciclos de Mejora en el Aula (2020). Experiencias de Innovación Docente de la US Esta obra se distribuye con la licencia Creative Commons 


\begin{tabular}{|c|c|c|c|}
\hline $\begin{array}{l}\text { Explicación de la } \\
\text { metodología del Role-Play } \\
\text { y su adaptación on-line } \\
\text { AC }\end{array}$ & $\begin{array}{l}\text { La profesora explica } \\
\text { cómo se realizará } \\
\text { la exposición } \\
\text { "teatralizada" } \\
\text { del caso clínico y } \\
\text { cómo se llevará a } \\
\text { cabo mediante la } \\
\text { plataforma }\end{array}$ & $\begin{array}{l}\text { Plataforma } \\
\text { Collaborate } \\
\text { Ultra }\end{array}$ & $10^{\prime}$ \\
\hline $\begin{array}{l}\text { Role-Play: Presentación de } \\
\text { los casos clínicos: } 3 \text { casos } \\
\text { del grupo A y } 3 \text { casos del } \\
\text { grupo B } \\
\text { AC }\end{array}$ & $\begin{array}{l}\text { Durante esta } \\
\text { actividad, tres } \\
\text { alumnos de cada } \\
\text { grupo realizan una } \\
\text { presentación del } \\
\text { caso clínico. Un } \\
\text { alumno hace el rol } \\
\text { de "dentista", otro } \\
\text { alumno hace el rol de } \\
\text { "paciente" y otro de } \\
\text { "profesor". }\end{array}$ & & $60^{\prime}$ \\
\hline $\begin{array}{l}\text { Puesta en común de } \\
\text { los role-play de cada } \\
\text { grupo Intervención del } \\
\text { profesorado } \\
\text { C }\end{array}$ & $\begin{array}{l}\text { El profesorado } \\
\text { comenta lo más } \\
\text { relevante de cada } \\
\text { caso clínico y el role- } \\
\text { play, resumiendo las } \\
\text { intervenciones y lo } \\
\text { más relevante }\end{array}$ & & $20^{\prime}$ \\
\hline
\end{tabular}

SESIÓN 4: DOCENCIA-CRÉDITOS PRECLÍNICOS. 26 noviembre 2020

\begin{tabular}{|l|l|l|l|}
\hline $\begin{array}{l}\text { DESCRIPCIÓN } \\
\text { FASE DEL MODELO METOD. }\end{array}$ & FINALIDAD & RECURSOS & T \\
\hline $\begin{array}{l}\text { Roll-Play: Presentación de } \\
\text { los casos clínicos: 3 casos } \\
\text { del grupo A y 3 casos del } \\
\text { grupo B }\end{array}$ & $\begin{array}{l}\text { Durante esta actividad, } \\
\text { tres alumnos de } \\
\text { cada grupo realizan } \\
\text { una presentación } \\
\text { del caso clínico. Un } \\
\text { alumno hace el rol } \\
\text { de "dentista", otro } \\
\text { alumno hace el rol de } \\
\text { "paciente" y otro de } \\
\text { "profesor" }\end{array}$ & & \\
\hline
\end{tabular}

Ciclos de Mejora en el Aula (2020). Experiencias de Innovación Docente de la US Esta obra se distribuye con la licencia Creative Commons 


\begin{tabular}{|l|l|l|l|}
\hline $\begin{array}{l}\text { Puesta en común de } \\
\text { los role-play de cada } \\
\text { grupo Intervención del } \\
\text { profesorado } \\
\text { C }\end{array}$ & $\begin{array}{l}\text { El profesorado } \\
\text { comenta lo más } \\
\text { relevante de cada } \\
\text { caso clínico y del role- } \\
\text { play, resumiendo las } \\
\text { intervenciones y lo } \\
\text { más relevante }\end{array}$ & 20' & \\
\hline $\begin{array}{l}\text { Rellenar individualmente } \\
\text { un cuestionario final con 6 6 } \\
\text { preguntas del tema sobre } \\
\text { el que se ha desarrollado } \\
\text { en el CIMA } \\
\text { C }\end{array}$ & $\begin{array}{l}\text { Conocer las ideas que } \\
\text { tienen los alumnos } \\
\text { sobre el tema que se } \\
\text { ha impartido }\end{array}$ & $\begin{array}{l}\text { Cuestionario } \\
\text { de Ideas } \\
\text { finales }\end{array}$ & $10^{\prime}$ \\
& & & \\
\hline
\end{tabular}

SESIÓN 5: DOCENCIA-CRÉDITOS CLÍNICOS. 12, 19 y 26 noviembre 2020

\begin{tabular}{|l|l|l|c|}
\hline $\begin{array}{l}\text { DESCRIPCIÓN } \\
\text { FASE DEL MODELO METOD. }\end{array}$ & FINALIDAD & RECURSOS & T \\
\hline $\begin{array}{l}\text { Realizar la exploración } \\
\text { clínica odontológica por } \\
\text { pares (entre alumnos) } \\
\text { AC }\end{array}$ & $\begin{array}{l}\text { El alumno deberá } \\
\text { realizar la exploración } \\
\text { odontológica a otro } \\
\text { alumno, aplicando } \\
\text { todas las nuevos } \\
\text { protocolos por la } \\
\text { Covid-19 }\end{array}$ & $\begin{array}{l}\text { Material de } \\
\text { exploración } \\
\text { clínica } \\
\text { EPls de } \\
\text { protección }\end{array}$ & $120^{\prime}$ \\
\hline $\begin{array}{l}\text { Realizar la historia clínica } \\
\text { y la exploración clínica } \\
\text { odontológica a pacientes } \\
\text { con patología sistémica } \\
\text { AC }\end{array}$ & $\begin{array}{l}\text { El alumno deberá } \\
\text { realizar la historia } \\
\text { clínica y la exploración } \\
\text { odontológica al } \\
\text { paciente con patología } \\
\text { sistémica, aplicando } \\
\text { todos las nuevos } \\
\text { protocolos por la } \\
\text { Covid-19 }\end{array}$ & $\begin{array}{l}\text { Material de } \\
\text { exploración } \\
\text { clica } \\
\text { protección de }\end{array}$ & $120^{\prime}$ \\
\hline
\end{tabular}

\section{Cuestionario inicial y final}

Es necesario conocer las ideas previas del alumnado y sus esquemas pre-concebidos sobre el contenido que queremos trabajar. Se seleccionaron las siguientes preguntas a partir de un caso clínico de urgencia odontológica frecuente y cercano:

Ciclos de Mejora en el Aula (2020). Experiencias de Innovación Docente de la US Esta obra se distribuye con la licencia Creative Commons 
María es una alumna que está estudiando en la actualidad en la Universidad de Sevilla el Grado en Derecho, segundo curso y se queja de un "un dolor de muelas" por una caries y acude a la Facultad de Odontología para que la trates.

1. ¿Cómo puedes llegar al convencimiento de que lo que tiene María se trata de una caries?

2. Una vez que ya le has preguntado a María por todo lo relativo a su muela, ¿qué otras preguntas de la anamnesis tendrías que preguntarle?

3. María dice que le has hecho una historia clínica muy completa. ¿A qué se refiere María?

4. Antes de que María llegase a tu consulta ha pasado por el triaje telefónico impuesto por la actual pandemia de la Covid-19 ¿sabes qué le han preguntado? ¿qué opinas al respecto?

5. María acude porque tiene "un dolor de muelas". ¿Qué exploración le harías como dentista para saber qué le pasa a la muela de María?

6. Como en la actualidad estamos inmersos en la pandemia de la Covid-19: ¿qué EPIS tienes que utilizar como dentista que examina y trata a María?

\section{Aplicación del ciclo de mejora en el aula}

\section{Relato resumido de las sesiones}

Sesión 1: Comienza la aplicación del CIMA el 22 de octubre del 2020, en plena pandemia de la Covid-19. La docencia que se impartió no formó parte del contenido teórico del CIMA. Al finalizar la clase, la profesora le explicó el cuestionario que recibirían por correo electrónico y se les envió a los delegados el Cuestionario inicial que tenían que rellenar individualmente. Cuestionario de 6 preguntas abiertas del tema sobre el que se desarrolló el CIMA Ciclos de Mejora en el Aula (2020). Experiencias de Innovación Docente de la US
Esta obra se distribuye con la licencia Creative Commons 
(Historia y Exploración Clínica en Estomatología y Patología Sistémica). El alumno lo contesta y lo envía por correo electrónico de nuevo a la profesora durante la hora de la clase. Se le indica al alumno que dicho cuestionario no tendrá calificación y que es importante que lo conteste sin consultar ninguna documentación.

La primera dificultad sobrevenida fue la no presencialidad de las clases teóricas y preclínicas. La explicación del cuestionario se realizó on-line, mediante la plataforma Blackboard Collaborative Ultra. Se ha experimentado la dificultad de la administración y recepción de los cuestionarios por vía telemática, por el elevado número de alumnos.

Sesión previa: El 29 de octubre del 2020. La he denominado sesión previa, porque la docencia que se impartió no formó parte del contenido teórico del CIMA, pero la metodología docente (trabajo colaborativo en grupos pequeños) que se diseñó fue el punto de partida para poder llevar a cabo la semana siguiente el ciclo de mejora, y es por ello que se ha querido resaltar la importancia de experimentar previamente la metodología docente. Durante la docencia preclínica del grupo A en horario de 8:00-10:00 y del grupo B en horario de 12:00-14:00, mediante la plataforma Blackboard Collaborate Ultra, se realizaron varios grupos de trabajo para que los alumnos aprendieran la dinámica del trabajo on-line mediante la plataforma educativa. Para que los grupos puedan funcionar y el alumnado sea capaz de trabajar en grupos, hay que formarlos previamente y practicar lo aprendido.

Hay que destacar que precisamente el día 29 de octubre, la plataforma sufrió varias incidencias técnicas (tanto por la mañana como por la tarde), se desconectaba y había que estar todo el tiempo reconectándose. Fue una experiencia muy enriquecedora, porque al tener la clase de 98 alumnos dividida en dos grupos, nos permitió desarrollar 
los grupos según las incidencias que ocurrieron. Cuando la conexión falló, como los alumnos ya conocían quiénes eran los miembros de cada grupo, ellos se organizaron por grupos de Whatsapp o Google y fue una grata sorpresa que, una vez finalizada la clase, pudieran entregar los trabajos. También hay que destacar la labor cooperadora de los delegados de curso al ser el nexo de unión entre el profesorado y los compañeros.

A pesar de las incidencias, se les consultó a los alumnos si querían trabajar en grupos mediante la plataforma Blackboard Collaborate Ultra y la mayoría asintió. También hay que resaltar que para utilizar estas herramientas online ha sido necesario que el profesorado asista y se forme en estas habilidades.

Sesión 2: Día 5 de noviembre de 2020. Comienza la docencia del tema Historia y Exploración Clínica en Estomatología y Patología Sistémica que hemos seleccionado para desarrollar el CIMA. La docencia de los créditos preclínicos del grupo A se ha realizado en horario de 8:00-10:00 y la del grupo B en horario de 12:00-14:00, mediante la plataforma Blackboard Collaborate Ultra.

- Planteamiento de casos problemas: La profesora presenta seis casos clínicos con imágenes clínicas, algunas radiografías y muy pocos datos médicos/ odontológicos para que los alumnos en grupos pequeños puedan "crearlos" y realizar un diagnóstico de la patología que presentan, tanto médica como odontológica. Con esta actividad se pretende enganchar y motivar a los estudiantes con casos que resulten de su interés (Bain,2007), creando un entorno para el aprendizaje crítico natural. Durante 10 minutos se presentan los seis casos clínicos y se dan las normas por escrito para desarrollar la actividad y la docencia. Se les envía por correo electrónico a los delegados y se suben a la plataforma. 
- Trabajo en Grupo: Idea de Alumnos: Cada Grupo de alumnos ( $\mathrm{A}$ y $B$ ) se subdividió en 6 grupos de 8 alumnos y se trabajó en la plataforma Collaborate Ultra.

Durante esta actividad el alumno realizó una descripción del caso clínico, así como la historia clínica y la exploración. Se le indica al alumno que debe ser creativo. Cada grupo de 8 alumnos realiza un informe escrito que lo entrega por mail al finalizar la sesión. La escritura como vehículo del proceso de enseñanza-aprendizaje (Finkel, 2008). La duración de la actividad fue de hora y media

- Resolución de dudas por parte del profesorado de la asignatura. El profesorado va ayudando a los diferentes grupos de alumnos durante toda la sesión, aclarando dudas y orientándolos. Va entrando en cada grupo de la plataforma. Durante las dos sesiones de hora y media de cada grupo A y B, se está colaborando con la dinámica de los grupos, con la parte técnica informática y con el contenido teórico y práctico.

Sesiones 3 y 4: Se realizaron los días 12 y 26 de noviembre de 2020.

- Trabajo en Grupo: Idea de Alumnos. Cada grupo de alumnos ( $\mathrm{A}$ y $B$ ) se subdividió en 6 grupos de 8 alumnos y se trabajó en la plataforma Collaborate Ultra.

- Puesta en común de los informes de cada grupo.Intervención del alumnado. El portavoz de cada grupo expone resumidamente el caso clínico con las aportaciones de mayor relevancia.

La exposición se realiza mediante "role-play" en esta ocasión y, motivado por el escenario B de docencia académica por la Covid-19, con carácter totalmente on-line. No quisimos renunciar a esta metodología docente por el hecho de no poder estar presentes en el aula. Y lo llevamos a cabo de manera virtual. Un alumno/a hizo el rol de Ciclos de Mejora en el Aula (2020). Experiencias de Innovación Docente de la US 
dentista, otro de paciente y otro de profesor/a. En esta sesión se presentan tres de los seis casos del grupo A y tres de los siete casos del grupo B.

La aplicación del "role-play" o simulación al campo de la Medicina permite la transmisión de conocimientos teóricos y prácticos (Levine y McGuire, 1970). La docencia de la competencia "saber diagnosticar" es adecuada para la realización de la metodología de la simulación.

- Puesta en común de los informes de cada grupo.

- Intervención del profesorado.

Cada profesora comenta lo más relevante de cada caso clínico y de los informes y se resumen todas las intervenciones y lo más destacable.

- El alumnado rellenó individualmente un Cuestionario de ideas finales con 6 preguntas del tema sobre el que se ha desarrollado el CIMA. El cuestionario se envió y se contestó por correo electrónico. Se pretendió conocer las ideas que tenían los alumnos sobre el tema que se había impartido con el desarrollo del ciclo de mejora.

Sesión 5: Días 12, 19 y 26 de noviembre de 2020. Comienza la docencia clínica presencial de ambos grupos de alumnos. Por la pandemia de la Covid-19 la presencialidad de los alumnos se realiza en grupos reducidos.

- Trabajo en grupo: Exploración clínica odontológica por pares (entre alumnos).

El alumno deberá realizar la exploración odontológica a otro alumno, aplicando todos los nuevos protocolos por la Covid-19. La docencia clínica permite al alumno desarrollar las habilidades aprendidas conceptualmente en la docencia teórica y preclínica.

Ciclos de Mejora en el Aula (2020). Experiencias de Innovación Docente de la US Esta obra se distribuye con la licencia Creative Commons 
Cada grupo de alumnos ( $\mathrm{A}$ y B) se subdividió en subgrupos de 2 alumnos y se trabajó en la clínica de la Facultad de Odontología. Antes de la pandemia esta práctica clínica la desarrollaba todo el grupo a la vez, pero ahora nos hemos adaptado a las circunstancias posibles y los alumnos van rotando y es por ello que se llevará a cabo durante cuatro o cinco días.

Los alumnos también tienen la oportunidad de conocer el material de exploración y los EPIs de protección clínica. Se insiste en ello, los tienen en códigos QR, el Procedimiento de Seguridad y Salud para la continuidad del trabajo y la actividad académica ante la pandemia Covid-19 del SEPRUS de la US y la Guía Preventiva específica ante el Covid19 para la Facultad de Odontología

- Trabajo en grupo: Exploración clínica odontológica a pacientes con patología sistémica. El alumno debía realizar la historia clínica y la exploración odontológica al paciente con patología sistémica, aplicando todas los nuevos protocolos por la Covid-19.

El principal obstáculo de enseñanza y de aprendizaje que ha marcado todo el desarrollo del CIMA ha sido la brusca adaptación a la modalidad on-line de los contenidos de las asignaturas, motivados por la situación actual sanitaria por la pandemia Covid-19. Esta situación sobrevenida ha obligado al desarrollo on-line de todas las actividades teóricas y preclínicas y, a la hora de desarrollar la práctica clínica con pacientes, nos hemos visto muy limitados en el número de alumnos y las nuevas medidas y protocolos.

Ciclos de Mejora en el Aula (2020). Experiencias de Innovación Docente de la US Esta obra se distribuye con la licencia Creative Commons 


\section{Evaluación del aprendizaje de los estudiantes}

Las contestaciones de cada pregunta del cuestionario inicial se categorizaron en los siguientes niveles: A (no tienen el conocimiento), B (conocimiento incompleto e incorrecto), C (conocimiento incompleto y correcto) y D (conocimiento completo y correcto).

Todas las preguntas han mostrado una tendencia bastante positiva en la mejoría del aprendizaje del alumnado.

Hay que destacar la pregunta 2 (Una vez que ya le has preguntado a María por todo lo relativo a su muela, ¿qué otras preguntas de la anamnesis tendrías que preguntarle?). La valoración de la contestación de esta pregunta se ha categorizado en varios niveles:

A- No tiene el conocimiento

B- Patología sistémica, patología, antecedentes personales

C- Patología sistémica, patología, antecedentes personales y antecedentes

D- Patología sistémica, patología, antecedentes personales y antecedentes y fármacos y alergias

En el cuestionario inicial solamente un 5\% del alumnado carece de conocimientos sobre la pregunta realizada. La contestación completa y correcta la realiza el 35\% del alumnado. El 85\% del alumnado presenta un nivel alto (C y D). En el cuestionario final aumenta hasta un $90 \%$ el nivel alto (niveles C y D), correspondiendo el 75\% al nivel D.

Ciclos de Mejora en el Aula (2020). Experiencias de Innovación Docente de la US Esta obra se distribuye con la licencia Creative Commons 


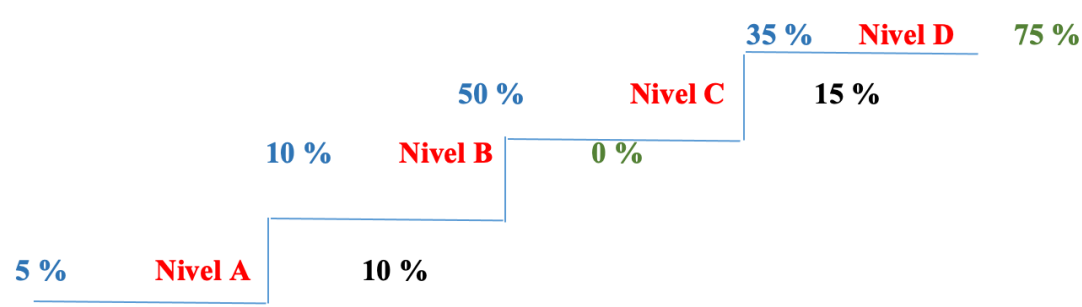

Figura 3. Escalera de aprendizaje correspondiente a la pregunta 2.

Y también hay que destacar la pregunta 4 (Antes de que María llegase a tu consulta ha pasado por el triaje telefónico impuesto por la actual pandemia de la Covid-19: ¿sabes qué le han preguntado? ¿qué opinas al respecto?). La valoración de las contestaciones de esta pregunta se ha categorizado en los siguientes niveles:

A- Triaje pandemia de la Covid-19 incompleto, faltan tres preguntas

B- Triaje pandemia de la Covid-19 incompleto, faltan dos preguntas

C- Triaje pandemia de la Covid-19 incompleto, falta una pregunta

D- Triaje pandemia de la Covid-19 completo

En el cuestionario inicial, solamente el 5\% del alumnado conoce el triaje telefónico de la pandemia de la Covid-19 completo y el 50\% de manera incompleta faltando una pregunta. En el cuestionario final se reduce a un 10\% los alumnos con menor conocimiento (el nivel $A$ ) y se incrementan hasta el 70\% los niveles superiores (C y D).

Ciclos de Mejora en el Aula (2020). Experiencias de Innovación Docente de la US Esta obra se distribuye con la licencia Creative Commons 


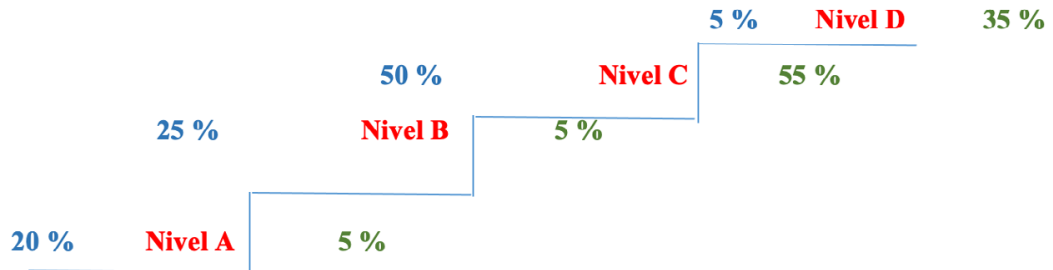

Figura 4. Escalera de aprendizaje correspondiente a la pregunta 4.

La comparación de los conocimientos adquiridos atendiendo a las respuestas aportadas a los cuestionarios inicial y final se muestra en la Figura 5. En el cuestionario inicial, un 13 \% del alumnado carece de conocimientos sobre las preguntas realizadas, siendo el nivel A el más inferior. El mayor porcentaje de alumnos se encuentra en el nivel C, representado por un $40 \%$; solamente el $26 \%$ contesta con la mayor corrección que viene representado en el nivel $D$.

En el cuestionario final se reduce a un $6 \%$ los alumnos con menor conocimiento (el nivel A) y se incrementa hasta un $42 \%$ el nivel más elevado (D).

\section{ESCALERA INICIAL}

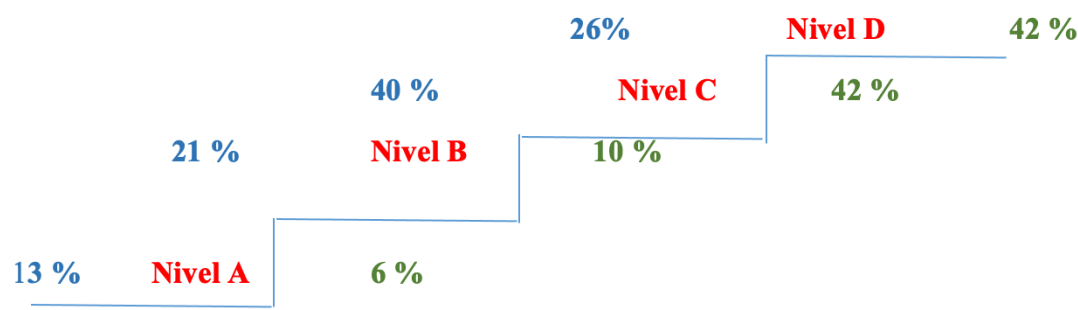

Figura 5. Escalera de aprendizaje cuestionario inicial y final.

Ciclos de Mejora en el Aula (2020). Experiencias de Innovación Docente de la US Esta obra se distribuye con la licencia Creative Commons 


\section{Evaluación del Ciclo de Mejora en el Aula}

A nivel cuantitativo, el Ciclo de Mejora ha presentado una evaluación bastante positiva al haber mejorado el aprendizaje del alumnado con respecto al cuestionario inicial en todas las preguntas.

Desde el punto de vista cualitativo, tanto por mis percepciones, como por las del resto de profesores y la del alumnado, el ciclo de mejora ha funcionado excelentemente, puesto que se ha conseguido motivar al alumnado y que participase activamente en la búsqueda de respuestas, liderando la búsqueda de su propio conocimiento.

El modelo metodológico que se les ha propuesto a través del role-play on-line ha permitido trabajar las habilidades comunicativas de la relación paciente-odontólogo, y ha facilitado trabajar la empatía y la perspectiva social. Enseñar a través de la simulación clínica permite al alumnado imaginar, crear y escenificar situaciones clínicas simuladas con alto grado de realismo. La dramatización o representación teatral permite integrar los conocimientos teóricos en la práctica.

El modelo metodológico que se les ha propuesto a través del "role-playing" on-line permite trabajar las habilidades comunicativas de la relación paciente-odontólogo, y facilita la empatía y la perspectiva social. Enseñar a través de la simulación clínica permite al alumnado imaginar, crear y escenificar situaciones clínicas simuladas con alto grado de realismo. La dramatización o representación teatral permite integrar la teoría y la práctica.

Mi experiencia personal sobre la realización de este ciclo de mejora docente en las situaciones actuales que estamos viviendo y sufriendo ha sido una experiencia única,

Ciclos de Mejora en el Aula (2020). Experiencias de Innovación Docente de la US Esta obra se distribuye con la licencia Creative Commons 
inimaginable, que ha pasado por muchos altibajos, puesto que en muchas ocasiones era un reto que parecía insuperable (sin alumnos en el aula, con un número muy superior de alumnos matriculados, con menor número de profesores por bajas, contagios y/o aislamientos preventivos, con pocos conocimientos de la plataforma Blackboard Collaborate Ultra, con infinidad de normas y nuevos protocolos diarios, con mucho cansancio, estrés, miedos y ansiedades...). Y este reto se ha podido transformar en una gratificante oportunidad. Una oportunidad personal como docente para adaptarme de la manera más rápida posible, para ser capaz de mejorar mi docencia. Precisamente en estos momentos es cuando es más necesario el cambio metodológico del proceso de enseñanza-aprendizaje, y con esta mejora docente se ha podido conseguir que el alumnado se motive y pueda ser partícipe de su propio aprendizaje.

Tras la aplicación del presente ciclo de mejora y los resultados obtenidos, pienso seguir introduciendo el presente modelo metodológico en la asignatura EP Sistémica y pienso incorporar el mismo a toda la práctica docente en el próximo curso académico.

Aspectos de la experiencia que se pretenden incorporar a toda la práctica docente habitual:

- Continuar con la reflexión sobre los tipos de contenidos que imparto en mi proyecto docente, realizando una identificación de contenidos conceptuales, procedimentales y actitudinales. Al tratarse de una asignatura con contenidos teóricos, preclínicos y clínicos intentaría de nuevo impartir el contenido del tema en las diferentes modalidades de docencia, incidiendo en la identificación de los contenidos más relevantes. Y también insistiendo en el contenido actitudinal de humanización de la docencia.

Ciclos de Mejora en el Aula (2020). Experiencias de Innovación Docente de la US Esta obra se distribuye con la licencia Creative Commons 
- Continuar elaborando mapas de contenidos que permitan una rápida visualización de conceptos y su interrelación y ayuden a construir el conocimiento del alumnado.

- Continuar trabajando en grupos las Ideas de los Alumnos y la búsqueda de preguntas problemas y, para ello, se seleccionarán casos clínicos que sean relevantes y específicos para cada temática concreta.

- Continuar impartiendo los cuestionarios iniciales y finales como exploración de las ideas del alumnado

- Continuar trabajando con la metodología docente role-play on-line. Hemos podido comprobar cómo se puede trabajar mediante la plataforma educativa Blackboard Collaborate Ultra y cómo es posible la evaluación mediante rúbrica. La metodología docente role-play on-line es una excelente herramienta para trabajar los contenidos actitudinales en una asignatura con alto contenido diagnóstico como es la asignatura EP Sistémica.

Ciclos de Mejora en el Aula (2020). Experiencias de Innovación Docente de la US Esta obra se distribuye con la licencia Creative Commons 
Palabras clave: Estomatología y Patología Sistémica, Grado en Odontología, Docencia universitaria, Experimentación docente universitaria, Dinámica de Roles.

Keywords: Stomatology and Systemic Pathology, Dentristy Degree, University teaching, University teaching experimentation, Role-Playing

\section{Referencias bibliográficas}

Bain, K. (2007). Lo que hacen los mejores profesores universitarios. Valencia: Universitat de València.

Finkel, D. (2008). Dar clase con la boca cerrada. Valencia: Universitat de València.

Levine, H.G \& McGuire, C.H. (1970). The use of Role Playing to evaluate affective Skills in Medicine. Journal of Medical Education, 45, 700-705.

Porlán, R. (Coord.) (2017). Enseñanza universitaria. Cómo mejorarla. Madrid: Morata.

Ciclos de Mejora en el Aula (2020). Experiencias de Innovación Docente de la US Esta obra se distribuye con la licencia Creative Commons 